\title{
Exploring the space of cognitive architectures
}

\author{
PAT LANGLEY \\ Robotics Institute, Carnegie-Mellon University, Pittsburgh, Pennsylvania 15213
}

\begin{abstract}
Production systems have been proposed as a candidate for the basic architecture of the human information processor. We consider a number of dimensions along which production systems can vary, and we discuss PRISM, a production system formalism designed to aid cognitive modelers in their exploration of the space of architectures. Examples of PRISM settings are presented in the context of particular models. Finally, some heuristics are suggested for efficiently searching the space of possible architectures.
\end{abstract}

Most branches of science begin by making observations and constructing taxonomies, but eventually they develop theories to explain those observations. Neches (1982), Newell and Simon (1972), and others have argued that the theories of cognitive psychology should take the form of running computer programs, or cognitive simulations. These same researchers have proposed production systems as the proper formalism for stating such theories, and an impressive number of production system simulations have now been constructed and tested. However, many different production system architectures are possible, and the cognitive simulator is faced with some choices when devising his models. In this paper, we consider the set of possible production system architectures, along with PRISM (Langley \& Neches, Note 1), a programming language designed to aid in the systematic exploration of these architectures. Before discussing the characteristics of production systems in general and of PRISM in particular, we should consider the goal of the cognitive modeler and the task with which he is concerned. In these preliminaries, we shall use a notion from cognitive psychology and artificial intelligence, that of a problem space.

\section{SCIENCE AS SEARCH}

One of the central insights of cognitive science is that intelligence involves search. Although this approach has been applied predominantly in explaining problem solving behavior, Newell (1980) has argued that it can be applied to a much broader class of intelligent action. Since the concept of mental search is based on an analogy with physical search, in which one moves through a physical space, one can view the problem solver as moving through some problem space in search of a solution. Effective search of a problem space requires some means of moving through that space in order to visit different locations. While the set of possible locations, or states, may be specified explicitly at the outset, it is much more common to define the space implicitly in terms of a generator that lets the problem solver move from one state to another. Given the ability to visit different states, one also requires a test to determine whether a particular state is equivalent to the goal state. However, while the test is necessary if one is to know when to stop searching, it is the generator that actually defines the space within which the problem solver moves.

The scientific process is an activity that fits very well into this search paradigm. In his attempt to formulate general theories, the scientist may be viewed as searching through a space of laws or hypotheses. In this domain, the generator outputs theories that are intended to explain some set of data. Once a theory has been proposed, it is tested against observations and tentatively accepted, modified, or rejected. One can view Kuhn's (1962) notion of paradigmatic revolutions as resulting from changes in the hypothesis space being searched. Simon (1973) has elaborated this view of science as search, arguing that the similarities between discovery and problem solving are much greater than their differences. Simon, Langley, and Bradshaw (1981) have suggested that an important component of discovery is the search for invariants, and they have implemented a model of this process in their BACON system.

As a branch of science, psychology should fit into this view of the discovery process. The research psychologist traverses a space of hypotheses, searching for one that will explain human behavior in some general way. If we assume this view is basically accurate, then it is natural to consider the form taken by the invariants of psychological theories. In the case of human behavior, an invariant is some aspect that remains the same across subjects, across task domains, and over time. We shall call the group of all such invariant features the cognitive architecture of the human information processing system. One of the major tasks of the cognitive psychologist, then (although by no means the only one), is to search the space of possible cognitive architectures and attempt to determine the particular architecture that is the basis of human behavior. 
Many alternate architectures are conceivable, and many different proposals have been made for the human architecture, ranging from neuron models to energy fields. However, 25 years of research in the information processing paradigm has greatly narrowed the class of acceptable solutions. One particular class of architectures, known as production systems, has shown great potential for explaining a wide range of cognitive phenomena. Newell and Simon (1972) were the first to outline the class of production system architectures and to provide arguments for their adequacy (which we will not recount here). However, this framework has since been tested by cognitive modelers in a wide variety of domains, ranging from problem solving (Rychener, Note 2) and natural language understanding (Anderson, Kline, \& Lewis, 1977) to memory tasks (Newell, 1973) and cognitive development (Klahr \& Wallace, 1976). In the following section, we review the basic assumptions of the production system approach and discuss the dimensions along which production system architectures can vary. These dimensions are discussed in the context of PRISM, a cognitive modeling language that can be used to explore alternate architectures.

\section{DIMENSIONS OF PRODUCTION SYSTEM ARCHITECTURES}

Structurally, production systems consist of a set of condition-action rules called productions and a dynamic work space called working memory. Some architectures also include a separate declarative long-term memory. However, all production system architectures share the first two of the above components, so we will focus on them here. Production memory and working memory are related through the recognize-act cycle, the basic mode of operation for production system architectures. During a cycle, the system first finds all productions whose conditions match elements in working memory. Given this set of matches, the system then selects one or more for application. The actions associated with these productions are then carried out, affecting working memory and causing new productions to match. A new cycle begins, and the process is repeated until no productions are matched or a halt command is encountered. Obviously, this account ignores many of the details, as well as the many variations that are possible within the basic framework. In this section, we expand on the steps in the recognize-act cycle and discuss some of the possibilities that occur at each stage.

PRISM (Langley \& Neches, Note 1) is a formalism for specifying production system architectures and for writing production system programs within those architectures. ${ }^{1}$ The PRISM language has been strongly influenced by Anderson, Kline, and Beasley's (1980) ACTF and by Forgy's (Note 3) OPS4, and it contains features from both languages. However, PRISM moves far beyond these earlier languages in its ability to specify

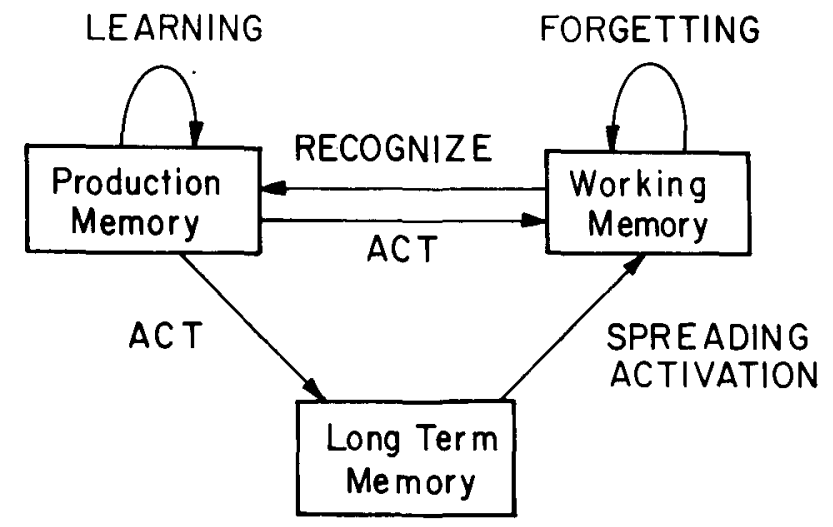

Figure 1. The components of PRISM and their interactions.

alternate architectures. This flexibility is accomplished through some 30 user-modifiable parameters. Some of these parameters take on numerical values; others accept the names of complex functions as their values. Since PRISM is implemented in LISP, the user may also define his own functions and use these as parameter settings. Taken together, these parameters and their possible settings define the space of production system architectures that can be stated within PRISM. In fact, one can view PRISM as a generator for this set of architectures. We have chosen to organize the following discussions around PRISM and its parameters. Figure 1 presents an overview of PRISM's components and the ways in which they interact.

\section{The Recognition Stage}

The first step in the recognize-act cycle involves determining which productions match against the current state of working memory. In discussing the matching process, it is important to realize that a production may match against working memory in more than one way. In fact, the same rule may even match against the same set of elements in more than one way. Each such match is called an instantiation, and the set of all true instantiations is called the conflict set. For this set to be computed, the architecture must have some notion of what distinguishes a "successful" match from an "unsuccessful" match. In this context, there are two basic possibilities: The system may consider only "complete" matches between productions and memory, or it may consider "partial" matches as well.

The first of these alternatives is simpler both conceptually and computationally. We say that a production matches completely against working memory if, for every condition in the production, there exists an element in working memory that maps against that condition, and further, that variables shared across conditions are bound in a consistent manner. This approach is simple corlceptually because one does not have to worry about degrees of matching; a production either matches completely or it does not match at all. The 
approach is simple computationally because if one encounters an unsatisfied condition while attempting to match a rule, the entire instantiation can be abandoned without examining the remaining conditions.

The vast majority of implemented production system architectures embody complete-matching algorithms. A major reason for this is the pioneering work carried out by Forgy (Note 4 ) on efficient techniques for computing complete matches. Earlier approaches simply went through a list of productions, comparing the conditions of each rule in turn to the contents of memory. In addition to being very slow, these systems took much longer to find instantiations of productions toward the end of the list. As a result, it was natural to try to save computing time by selecting the first match found for application, so that in these early architectures, the recognition and conflict resolution stages were inherently intertwined. Forgy's technique takes advantage of structural redundancies in the conditions of productions, as well as temporal redundancies in working memory (which changes slowly over time) to compute all true instantiations of the rule set in a reasonable time. PRISM takes advantage of Forgy's matching technique, and as we shall see below, this allows great flexibility in the conflict resolution stage.

Before moving on, we should say a few words about the partial matching of productions. As its name implies, this process means that one allows instantiations into the conflict set that do not satisfy all of the conditions of some production, or whose variables do not bind in a consistent manner. Anderson (1983) has explored the former type of partial matching system, and Kline (1981) has studied the latter type. The problem here is twofold. First, it is not clear just which partial matches should be allowed into the conflict set, or what should be done with them should they be selected for firing (for example, the action side may then be incompletely determined). Second, if the computation of all complete matches is difficult, the computation of all partial matches is much more difficult. To build running simulations based on partial matching notions, we will most likely have to be satisfied with techniques that find only some of the many possible partial matches. For example, Anderson (1983) has explored architectures that can compute a subset of all partial matches, which is determined by the manner in which rules are stored. Hayes-Roth (1978) has suggested we abandon algorithmic approaches to computing partial matches and explore heuristic search approaches that would output only the "best" partial matches. Despite its associated difficulties, the notion of partial matches is a promising one that is beginning to attract considerable attention among cognitive simulators. However, since complete matching systems are much more fully understood than their partial matching counterparts, we will focus on the former in the remainder of the paper.

\section{Conflict Resolution}

Once the production system has computed all true instantiations, it must decide which of these instantiations should be selected for application. The set of true instantiations makes up the conflict set, and the selection process is called conflict resolution. McDermott and Forgy (1978) have discussed alternate strategies for conflict resolution and their implications for production system behavior. Early production system languages (e.g., Waterman, 1975) simply selected the first instantiation encountered by the matcher, whereas more recent languages have shifted to other criteria, such as recency (Forgy, Note 4) and specificity (Anderson et al., 1980). PRISM divides the conflict resolution process into three separate dimensions, each of which can take on a number of values. These dimensions include the following:

Refraction-In some cases, the modeler may desire to have instantiations removed from the conflict set permanently. This process is called refraction, and it can be implemented in a number of ways in PRISM. For example, one can remove only those instantiations that were applied on the previous cycle; this is useful in avoiding loops in which the same production applies time after time, and it has been used extensively in the OPS languages (Forgy, Note 3, Note 4). Alternately, one can remove all instantiations in the conflict set, considering for selection only those that have become true on the current cycle; this approach tends to focus attention on recent events and has been used in some versions of ACT (Anderson et al., 1980). Finally, one can avoid refraction entirely, making no permanent changes to the conflict set.

Ordering-Many instantiations may still remain in the conflict set after refraction has occurred, and before one can select among them, they must be ordered along one or more dimensions. The list of PRISM options includes the strength of rules, the recency of the true productions, the summed activation of the matched working memory elements, and random ordering. Other ordering rules can be easily defined in LISP.

Selection-Once instantiations have been ordered, various strategies remain for selecting among them. For example, one might want to select all those instantiations matching against rules with the highest strength and then select randomly from among the remaining instantiations with the highest summed activations. Another option is to reject each instantiation whose value falls below a threshold on some dimension and to apply the remainder in parallel. Combinations of these two basic strategies are also possible, and one can again easily define new selection strategies in LISP.

Taken together, the options available for these three aspects of conflict resolution give the PRISM user considerable control over the behavior of his production system models. For example, a few keystrokes would suffice to alter a serial program based on production 
strength to a parallel one based on activation levels. We believe such flexibility is a definite asset in efficiently exploring the space of production system architectures.

\section{The Act Stage}

Once a set of instantiations has been selected for application, the actions of the relevant productions are retrieved and their action sides are instantiated as well. These instantiated actions are then carried out, usually affecting working memory. The most commonly used actions involve the addition and deletion of working memory elements, but a production may also add or remove things from a long-term propositional memory, retrieve things from that memory through a process of spreading activation, or learn through the creation of new productions or the strengthening of existing ones. Although the last two actions may be technically included in the act stage, they are important enough to be treated separately, and we have done so in later sections.

Since some architectures examine the activation of working memory elements during conflict resolution and other stages, the activation given to an element when it is first added to memory takes on some importance. PRISM allows the user to specify a default initial activation for working memory elements, and this activation is given to all elements unless otherwise specified. In particular productions, the user may also specify the activation that should be given to a particular element when that production is applied. In both cases, if an element is added that is already present in working memory, the old and new activations are added together.

PRISM's main flexibility during the act stage comes from a facility for specifying actions that should be carried out automatically during every production firing. These default actions may require no arguments, as when one automatically strengthens the production currently being applied. However, in other cases, one may wish to apply default actions to the instantiated structures in the right-hand side of a rule. For example, one might want every element that is added to working memory to also be added to the propositional network, or to spread activation from these elements to others already stored in the propositional net.

For example, if PRISM's default actions were set to $((<\mathrm{add}>\mathrm{x}))^{2}{ }^{2}$ it would behave much like Forgy's (Note 4) OPS2, adding the instantiated right-hand side elements only to working memory. However, if the default actions werc instead set to $((<\mathrm{add}\rangle \mathrm{x})(<\mathrm{add}$ to-net $>x)(<$ spread $>x)(<$ exercise $>))$, it would behave much more like Anderson et al.'s (1980) ACTF production system language, in that it would also store elements in the propositional network, spread activation from them, and strengthen the currently firing production. Thus, one can see that this facility provides for considerable variety in the behavior of the basic architecture.

\section{Forgetting}

The notion of forgetting is important to cognitive psychology, but to include it in our framework, we must introduce a fourth stage to the basic production system architecture. This stage includes two basic subprocesses: the decay of activation in working memory and the fading or removal of elements as the result of that decay. Since these mechanisms may be affected by events occurring during the act stage, we have delayed consideration of them until now.

There are two basic processes that can lead to decay. We will call these time-limited decay and space-limited decay, and both have been discussed in the literature on memory (Waugh \& Norman, 1965). In time-limited decay, the activation of elements in working memory decays at the same rate for each element, and entirely as some function of time. For example, during every cycle, the activation of each element might be multiplied by some factor. Other decay functions are possible, and PRISM will allow the use of any LISP defined mathematical function. And while decay normally occurs once every cycle, the user can force it to occur on every production firing through the default actions parameter. In space-limited decay, the total amount of activation is fixed to equal a user-controlled value. As new elements enter working memory, the activations of existing elements are normalized so that the total activation remains constant. Thus, activation tends to decrease with time, but the exact amount of decay depends on the number of elements entering memory and the initial activation of each.

The decay process can affect a production system's behavior during conflict resolution, provided the selection strategy is sensitive to the activations of working memory elements. As we have seen, one commonly used conflict resolution technique is to prefer instantiations matching against more active elements. However, when combined with a fading mechanism, decay can affect the nature of the conflict set itself. Every cycle, PRISM examines each working memory element to see if its activation has fallen below a user-specified threshold. ${ }^{3}$ When this occurs, the element is removed from working memory and forgotten. This in turn may lead to the removal of instantiations from the conflict set whose conditions matched against the forgotten element, or to the introduction of new instantiations whose negated conditions matched against the same element. Alternately, one may view such forgetting as simply another conflict resolution principle, in which the system refuses to select instantiations matching against elements that have fallen below a certain thresh. old.

\section{Spreading Activation}

The distińction between short-term and long-term memory leads naturally to the notion of retrieval. In order for retrieval to occur, elements that have faded or 
been deleted from working memory must be stored in some long-term memory. PRISM includes such a long. term store and allows elements to be inserted either automatically (through the default actions) or explicitly by the user or by the action side of a production. Each element in the long-term store has an associated trace strength, which plays a role in its retrieval. Parameters control the trace strength an element is given when it first enters long-term memory, as well as the manner in which this strength is increased when it is added at later times. While many forms of retrieval have been postulated, one of the most appealing proposals involves a process of spreading activation (Anderson, 1976). In this scheme, activation spreads from an element that is being inserted in working memory to other elements with which it is connected in some fashion. These connections usually involve shared symbols. For example, if the element (love john mary) is being added to memory, activation might spread to other elements in the long-term store that contain the symbols "love," "john," and "mary," such as (love mary joe). PRISM incorporates a spreading activation mechanism, and allows this process to be either automatic (through the default actions) or directed through particular working memory elements by the action side of a production.

PRISM provides two basic types of spreading activation mechanisms. The first, called spreading to a limit, involves the notion of decay as activation spreads farther from its source. In this approach, elements that are indirectly connected to a source element may still be activated, but they will receive proportionately less activation as the number of intermediate elements that must be traversed increases. The particular decay function involved can be specified by the user, as can the limit beyond which activation ceases to spread. In addition, activation is divided among elements connected to the source element; however, rather than being divided equally, each element is given activation proportional to its trace strength in long-term memory. The amount of activation to be spread from the source node is determined by a user-controlled parameter, unless it is otherwise specified in the action side of the production directing the spreading process. An alternative process involves spreading to a depth; in this approach, activation spreads equally to all elements in long-term memory that are within a certain distance from the source element. The depth to which activation spreads can be controlled by parameter or can be explicitly given in the right-hand side of a production. The amount of activation spread to each element is controlled by another PRISM parameter. The former of these methods bears a strong resemblance to Anderson's (1976) version of spreading activation, but it is much more expensive to compute than the second method.

\section{Learning}

One of the central arguments for considering production systems as the architecture of human cognition revolves around their modularity. Since production system programs are composed of relatively independent condition-action rules, it is easy to imagine (at least in the abstract) a production system that would continue to function if new rules were inserted or if existing rules were removed. Young (1976) has provided an example of the first type of system in the domain of seriation strategies, and Young and O'Shea (1981) have used the second approach to explain the occurrence of subtraction bugs. This modularity suggests that production system architectures would provide an ideal framework for modeling the incremental nature of much human learning and development, and a number of researchers have attempted to expand on this idea. Below, we consider PRISM's facilities for modeling learning phenomena.

The most straightforward way of creating new productions is through the process of designation. In this learning method, the general form of a production is specified in the action side of some rule. When this rule matches against a particular set of working memory elements, the variables in its condition side are bound in some way. These bindings are then carried over into the action side of the rule and used to construct a new production based on the general form by replacing the variables found in that form with their bindings. In its simplest version, the designating rule must know the exact number of conditions and actions in the new production, as well as their forms. However, designating rules can in principle interact with other rules for intelligently gathering such conditions and actions; in such cases, the designating rule must know nothing more than that productions consist of a set of conditions followed by a set of actions.

PRISM also has the ability to learn through generalization. In this process, productions that are isomorphic in structure or form are noted, and a more general rule is created by replacing differing constants with variables. ${ }^{4}$ The new rule is guaranteed to match whenever the two specific rules match, as well as in new situations. This approach is usually used in combination with the desig. nation process, with designation creating specific rules and generalization using these rules as evidence for introducing new rules with a broader range of applicability. PRISM allows the user to turn this facility on or off, since there are cases when he may not want the system to automatically introduce new rules into the system. In addition, the user can specify symbols that should never be generalized across. For example, given a working memory analog of a semantic network, one would not want to generalize across relations like "is-a" or "type." Such symbols are called syntax elements, since they fulfill the same function in working memory elements that prepositions and inflections perform in sentences.

The generalization method is complemented by an inverse process of discrimination. While generalization occurs automatically whenever two rules with identical forms are found, the discrimination mechanism must 
be explicitly evoked in the action side of a production. The discrimination technique is called when some error has been noted, and it attempts to modify the system so that this error does not occur in the future. The first step in this process is to retrieve the instantiation that led to the undesirable action. This "bad instance" of the responsible production is compared to the last "good instance" of the same production in order to find some difference between the two situations. Differences consist of elements or sets of elements that were present in working memory during one situation but not in the other. For each difference that is found, PRISM creates a variant of the faulty production that contains one or more additional conditions. ${ }^{5}$ These conditions guarantee that the variant would match in the desirable case, but not in the situation that led to an error. If a variant is still overly general, it will lead to new errors, and the discrimination process will be called again to generate even more conservative rules.

Since PRISM allows the strength of productions to play an important role during conflict resolution, mechanisms for altering the strength of productions play an equally important role in learning. PRISM allows the user to specify the precise function to be used when weakening or strengthening a rule. For example, one might decide to multiply the current strength by some fraction when weakening, but to add a constant term when strengthening. However, the exact functions used in modifying strengths are less important than the overall effect of strengthening and weakening. For example, PRISM automatically increases the strength of a production whenever it is relearned. This proves very useful for systems that learn by discrimination, since many of the variants generated during discrimination are based on spurious differences. By giving these variants low initial strengths and strengthening them whenever they are recreated, a system can focus its attention on variants that have been generated many times and, thus, are more likely to be correct. Similarly, it seems a good strategy to weaken rules that lead to errors, so that their variants can come to mask them more quickly. Strengthening can also be evoked explicitly in the action side of a production, if it is desirable to reward a rule for some activity it has performed. Also, productions can be set to strengthen themselves whenever they apply, so that a rule selected at one point will be even more likely to be selected in the future. As we have seen, a command to this effect can be placed in an architecture's default actions, leading to an exercise effect for the entire production system.

The above methods certainly do not exhaust the range of possible learning techniques applicable to production systems. For example, Lewis (Note 5) has discussed the composition of productions into larger rules. Similarly, Neves and Anderson (1981) have attempted to account for the transformation of declarative knowledge into procedural knowledge through a process of proceduralization. Other methods for constructing productions are certainly possible, and our understanding of this quadrant of the space of cognitive architectures is far from complete. However, we trust that the methods outlined above will provide some assistance in constructing models of human learning and development.

\section{SOME EXAMPLES OF PRODUCTION SYSTEM ARCHITECTURES}

Our discussion of production system architectures has so far been only in the abstract, and a few examples are in order. Since our discussion of the space of architectures has been interleaved with a discussion of PRISM, we will focus on models that have been implemented in this formalism (see Table 1). Below, we examine two related systems that are capable of learning search strategies from experience. These programs are not intended as detailed models of human performance, but they do reveal some interesting architectural design issues. After this, we discuss a production system model of the language acquisition process. Although learning systems have been chosen as examples, the main concern will be with aspects of their performance and with the influence of the architecture on that performance.

\section{Learning Search Strategies}

SAGE (Langley, 1982c, in press) is a model of strategy learning that is stated as a production system and implemented in PRISM. The program begins with a set of productions for proposing legal moves for some problem, and it uses these rules to find a solution path by trial and error. Once it knows the solution to a problem, SAGE uses this knowledge to assign credit to good applications of its rules and blame to bad applications. Using the discrimination process described above, the system constructs more conservative move-proposing rules that incorporate features of the good instances that did not occur in the bad instances. While the original rules contained only the legal conditions for application, these variants include heuristically useful conditions for directing search down profitable paths. These variant rules are given a low initial strength, but this is increased whenever the rule is relearned. SAGE has acquired useful search heuristics for the slide-jump puzzle, a seriation task, and algebra problems in one variable.

Let us examine some components of SAGE's architecture, along with their implications for the system's search strategy. The relevant components involve the conflict resolution and decay settings, which are:

$\begin{array}{ll}\text { refraction: } & \text { refract-fired } \\ \text { ordering: } & \text { strength activation random } \\ \text { selection: } & \text { best best best-one } \\ \text { decay-strategy: } & \text { time-decay } \\ \text { decay-function: } & (* \times 0.9)\end{array}$

Taken together, these settings lead SAGE to carry out a depth-first search through its problem space. The standard 
Table 1

Dimensions of PRISM Architectures

The Recognition Stage

Complete Matching, Partial Matching (not implemented)

The Conflict Resolution Stage:

Refraction: refract fired, refract all, refract none

Ordering: strength, motivation, production order, random

Selection: best, best one, all

The Act Stage

Default Actions: add-to-wm, add-to-ltm, spread, exercise, any function

Default Activation: any number

Default Trace Strength: any number

Trace Strengthening Function: any function

The Forgetting Stage

Decay Strategy: time decay, space decay

Time Decay Function: any function

Space Decay Total Activation: any number

WM Activation Limit: any number

Fade Time: any integer

Spreading Activation

Spread Strategy: spread to limit, spread to depth

Spread Limit: any number

Spread Limit Decay: any function

Spread Depth: any integer

Spread Depth Activation: any number

Learning

Learning Functions: designation, discrimination, generalization, strengthening, weakening

Default Production Strength: any number

Production Strengthening Function: any function

Production Weakening Function: any function

refraction setting lets the system avoid simple loops in which the same move is proposed again and again. The ordering and selection settings bias the program in favor of moves proposed by stronger rules; in general, rules that have been learned more often will have higher strengths and will thus tend to direct the search process. Among rules of equal strength, SAGE prefers those matching against more recent working memory elements. Since activation of such elements decreases with time (due to the decay-strategy and decay-function setting), this bias leads the system to focus on states that have just been created. Finally, if two or more instantiations have equal activations, SAGE selects one of these at random. The selection of a single instantiation leads to the creation of a single new state, and it is precisely this characteristic that distinguishes a depthfirst control structure from the alternatives. Also note that if SAGE encounters a dead-end state at which none of the move-proposing rules can be legally applied, the bias toward recency will automatically lead the system to back up to the previous state and continue the search from there. SAGE shares this depth-first control structure with a number of other recency-based production system architectures, such as Forgy's (Note 3) OPS4.

Although SAGE has learned useful search heuristics in three rather different domains, it has two serious drawbacks. First, SAGE's depth-first search strategy can never discover more than a single solution, even when multiple solution paths of equal length exist. In such cases, blame might be incorrectly assigned to a good move, confounding the learning mechanisms. Although its reliance on the strengthening process should let SAGE recover from such errors in time, they would cause complications and should be avoided if possible. Second, the system requires a complete solution path in order to apply its learning techniques. While this conservative approach simplifies the credit assignment problem, there are many domains in which blind search would simply take too long. In such cases, it is desirable to learn about the structure of the space as it is being searched, so that later stages of the search are more directed than earlier stages.

In response to these limitations, we have developed a new version of SAGE that carries out a modified breadthfirst search. The necessary settings are remarkably similar to those for the first version, but the system's behavior is radically different:

$\begin{array}{ll}\text { refraction: } & \text { refract-fired } \\ \text { ordering: } & \text { strength activation } \\ \text { selection: } & \text { best best } \\ \text { decay-strategy: } & \text { time-decay } \\ \text { decay-function: } & \left({ }^{*} \times 0.9\right)\end{array}$

In the new architecture, refraction is unchanged, and preference is still given to rules with higher strengths and to instantiations matching against more recent elements. However, once these dimensions have been used to filter out undesirable instantiations, the new 
SAGE does not bother to select one of these matches at random. Instead, it applies all of the remaining instantiations in parallel and makes the resulting moves simultaneously. In this scheme, SAGE may occasionally back up to an earlier state,${ }^{6}$ but by and large, the system proceeds by expanding a number of nodes at one level, then moving on to the next level, and so on until a solution is found. In this way, the program is able to find multiple solution paths and so avoid the blame assignment difficulties of the earlier version. In addition, the availability of many states and paths lets the new system discover regularities in a problem space before a solution has been reached. For example, it lets SAGE incorporate a variant of Anzai's (1978) shorter path heuristic, which notes when the same state has been reached by two paths of different lengths, and to learn rules that prefer the shorter route to the longer one. Such learning heuristics are especially useful in tasks like the Tower of Hanoi, in which the state space is very dense and multiple-move loops are possible.

While the learning aspects of SAGE are interesting and significant, our major concern here is with production system architectures. The striking feature of this example is that such a small change in the architecture can have such an important effect on behavior. The fact that such architectural alternatives can be so easily stated in the PRISM formalism testifies to its usefulness in exploring the space of possible cognitive control structures.

\section{Modeling First Language Acquisition}

First language learning is another area in which incremental learning seems to predominate, and we have used PRISM to construct AMBER (Langley, 1982a, $1982 \mathrm{~b}$ ), a model of the early stages of language acquisition. The model starts with the ability to say only one word at a time and learns by comparing its incomplete utterances to what an adult would say in the same situation. Like the strategy learning systems described above, AMBER modifies its speech behavior by creating new productions and strengthening old ones. When it makes an error of omission (by failing to predict an adult utterance), the model designates a production to say that word in the future. When AMBER makes an error of commission (by predicting a word or morpheme that should not occur), it evokes the discrimination process in order to discover the conditions under which the word should be spoken. The model accounts for some well known child language data, including the omission of content words and the order in which grammatical morphemes are mastered.

AMBER's architecture is interesting because it gives the model the potential for producing nearly complete sentences at the outset but forces the model to learn specific rules for this potential to be actualized.
Let us begin by examining AMBER's conflict resolution and decay settings:

$\begin{array}{ll}\text { refraction: } & \text { refract-fired } \\ \text { ordering: } & \text { strength activation random } \\ \text { selection: } & \text { best best best-one } \\ \text { decay-strategy: } & \text { time-decay } \\ \text { decay-function: } & (* \times 0.9)\end{array}$

These settings are identical to those for the first version of SAGE, and they lead to similar effects. However, while SAGE was concerned with searching problem spaces, AMBER's goal is to generate sentences, so the behavior of the two systems is not identical. In order to understand AMBER's potential and its initial limitations, we must examine its representation of sentence meanings and the rules that initially control the expression of those meanings.

AMBER's input consists of two pieces of information: the meaning to be expressed in a sentence and the main goal or topic to be expressed. Meanings generally correspond to some action or event and are represented as a set of working memory elements. For example, the sentence "John bounce ed the red ball" would be represented by elements like (action-1 agent agent-1), (action-1 action bounce), (action-1 object object-1), (agent-1 person John), (object-1 color red), and (object-1 type ball). In this formalism, tokens such as "action-1" and "agent-1" are connected to each other by relations like "agent." This information can be represented graphically, using nodes for tokens and labeled links for relations. Taken together, they form a connected graph or network that expresses the meaning of some event. However, there are still many ways this meaning can be expressed, and it is here that the main topic comes into play. The main topic is given as another working memory element, such as (describe action-1) or (describe agent-1). This information can be used to impose a tree structure on the network by specifying the top node in the structure. If the main goal were action-1, the tree shown in Figure 2 would result, and a sentence like "John bounce ed the red ball" would be appropriate. However, if

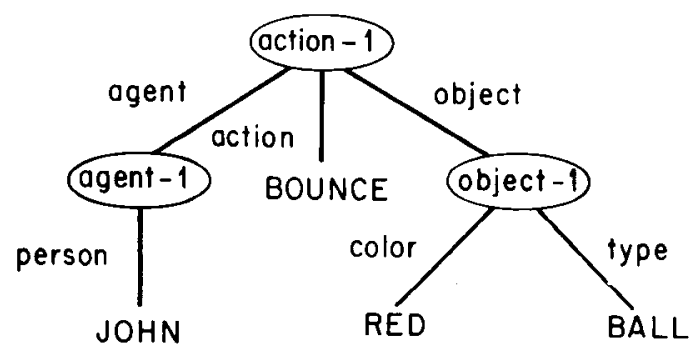

"Figure 2. An AMBER goal tree. 
agent-1 were instead the main goal, then a sentence like "It was John that bounce ed the red ball" would be correct.

AMBER is a goal-oriented system, and modified networks like that shown in Figure 2 are interpreted as goal trees. In generating a sentence, AMBER starts with a goal to describe the entire event. It then selects one of the nodes lower in the tree and sets up a subgoal to describe this part of the event. In order to carry out this scheme, AMBER requires one production for establishing subgoals, another for saying a word once a terminal node has been reached, and a final rule for marking goals as satisfied. These three productions (named "start," "speak," and "stop") are shown in Table 2, along with their relative strengths. Taken together, these rules give AMBER the potential for generating sentences containing all relevant content words. However, there are two problems with the behavior that results from these rules. First, no constraints are placed on the order in which subgoals are generated, so that object-1 would have as much chance of being selected first as would agent-1. Second and even more important, the stop rule is stronger than the start rule. This means that as soon as any goal has been marked as satisfied (as occurs whenever a word is said), all supergoals will be marked satisfied as well and the system will halt after uttering a single word. In this way, AMBER's conflict resolution strategies are used to explain the young child's potential for generating multiword utterances, but his inability to say more than one word at a time.

Based on errors of omission, AMBER constructs rules for setting up specific goals (such as describing the agent of an event) and for sequencing pairs of subgoals (such as describing the object after the agent has been described). These rules are created through the designation process, and they are strengthened whenever they are relearned. Upon exceeding the strength of the stop rule, they begin to affect AMBER's behavior and lead to multiword utterances. However, our concern

Table 2

AMBER's Productions for Manipulating Goals

START

If you want to describe nodel,

and node 2 is directly below nodel in the goal tree, then establish a goal to describe node 2 .

Strength: 10.0

SPEAK

If you want to describe concept, and word is the word for concept,

then mark the concept goal as satisfied, and say word.

Strength: 15.0

STOP

If you want to describe nodel,

and node 2 is directly below nodel in the goal tree,

and node 2 has been satisfied,

then mark nodel as satisfied.

Strength: 20.0 here is with architectures and their relation to behavior, so let us consider what would happen if the model's start rule had a higher strength than the stop rule. In this case, once a goal had been marked as satisfied, other goals would have a chance to be proposed. Given the architecture specified above, preference would be given to instantiations matching against more recent elements. Effectively, this means that an instantiation matching against the immediate supergoal would always be selected if possible, so that another goal at the same level as before would be established. After all goals at a given level had been satisfied, the system would back up a level and set up a subgoal at the next higher level.

For example, given the tree in Figure 2, this modified AMBER might first set the subgoal (describe agent-1), followed by (describe John). After saying the word "John," it would back up to the first level, where it might set the goal (describe object-1), followed by (describe ball). After saying the word "ball," it would then add (describe red), say the associated word, and back up again. Finally, the model would insert the goal (describe bounce), say "bounce," and halt. This is only one of many possible orders in which the goals might be established, but it is important to realize that certain orders would never occur. For example, sentences like "John ball red bounce" and "bounce red ball John" could occur, but a sentence like "John red bounce ball" would never be produced. The former sentences satisfy what Anderson (1977) has called the graph deformation condition, but the last sentence does not. This constraint states that if two concepts occur close together in the meaning of a sentence, then the associated words must occur close together in the sentence. As we have seen, AMBER is capable of generating (and thus learning to generate) utterances that obey this constraint, but not those sentences that violate it. Moreover, this bias results not from any special rules concerned with enforcing the graph deformation condition, but from a conflict resolution strategy that prefers recent elements to older ones. This appears to be an example in which architectural assumptions provide real explanatory power.

\section{CONCLUSION: SOME SEARCH HEURISTICS}

In the preceding pages, we have considered the space of production system architectures and a formalism for specifying them. We have examined a few of the architectures contained in this space and seen some ways they can be used to explain cognitive behavior. We hope we have convinced the reader that tools like PRISM have an important role to play in devising theories of cognition. However, simply specifying the space containing the human architecture is quite different from determining that architecture itself. It would be difficult to estimate the exact size of the space determined by PRISM's options, ${ }^{7}$ but there is little doubt that an unsystematic search would take a prohibitively long time. Clearly, then, the cognitive modeler must have some heuristics for directing search through this vast 
space, and it is appropriate to suggest a few such heuristics before closing.

Useful heuristics must take advantage of some regularity in the space to which they are applied, so it is appropriate to ask what sorts of regularities occur in the space of production system architectures. One sort of regularity involves interactions between dimensions. An obvious interaction occurs between the decay strategy used and the decay function and total activation parameters. The setting of the decay function affects behavior only when the decay strategy is set to time decay; similarly, the setting of total activation affects behavior only when the decay strategy is set to space decay. In other words, the space defined by PRISM's options has a hierarchical structure, so that the total number of possible architectures is not quite so large as it appears.

Other interactions exist that lead to "nonviable" architectures. For example, imagine a system that increased the activation of working memory elements on every cycle but still preferred instantiations that matched against elements with higher activations. Such a system would run, but because it would focus on events that occurred in the remote past, it is unlikely that we would find its behavior acceptable. Another example comes from our own experiences in constructing learning systems. When first exploring systems that learned by discrimination, we tried using a combined measure as our major ordering function during conflict resolution: We took the strength of each production, multiplied this value by the summed activation of the matched working memory elements, and selected the instantiation with the highest combined score. Now, the discrimination process sometimes created variants with a single new condition and other times created versions with two or more new conditions. At still other times, it built rules that contained negated conditions that could not match against any elements in memory. Given its conflict resolution strategy, our system always preferred variants with positive conditions to those with negative conditions (since the latter contributed nothing to the summed activation), and it always preferred variants with more conditions to those with fewer (assuming equal strengths). As a result, the system would enter a "learning loop" in which it would select the most complex variant for application; this would lead to an error, a more complex variant would be generated, this would be selected, and so on. The combination of discrimination and the strength-timesactivation scheme led to a nonviable learning system, and we eventually resorted to conflict resolution strategies like those shown for SAGE and AMBER, which lead to quite robust systems.

Another regularity in the space of architectures relates to parameters involving numbers, such as the production strengthening and weakening functions. In experiments with learning systems that use strengthening and weakening techniques to alter behavior. the relative values used in these parameters were more important than the absolute values. We would expect a similar situation to hold for other interacting numerical parameters, such as spread limit and the spread limit decay function. When such a relation is established, the effective dimensionality (and thus the size) of the search space is reduced, since one knows that certain architectures will lead to identical behavior despite their different settings.

A final heuristic that should aid the modeler's search involves the notion of a planning space. A planning space is an abstracted version of some problem space in which some differences have been ignored. As a result, there is a many-to-one mapping between the states in the original space and the simpler states in the planning space. This means that the new space is smaller and easier to search. Once a solution has been found in the planning space, this solution can be used as a plan to aid search in the original space. While there is no guarantee that this plan will lead to an actual solution, it can be of great aid in directing search. The application of this approach to the space of architectures is straightforward. In some domains, the modeler may not be concerned about the details of some of the cognitive dimensions discussed above. For example, in constructing SAGE and AMBER, we wanted the activations of working memory elements to decrease over time, but a time-based or a space-based decay strategy would have done equally well for our purposes. In fact, we choose a time-based strategy because it is computationally slightly less expensive, but the behavior of the system would have been unchanged if we had instead used a space-limited scheme. Thus, there are many cases in which a partial specification of the architecture is sufficient to produce the desired behavior. If this architecture is later applied to some new domain in which the previously irrelevant dimension comes into play, the architecture can be revised and further specified at that point. In this way, the cognitive modeler may temporarily focus his attention on major phenomena and defer his decision on other features until later.

We doubt that the above heuristics alone will enable the reader to search the space of architectures in an efficient manner. For that, he must also take advantage of his knowledge of cognitive phenomena, since the behaviors to be explained are the most important constraints one can place on a theory. For example, we drew on our knowledge of depth-first and breadth-first search strategies in devising the two SAGE architectures. And while explaining the graph deformation condition was not our main concern while we were planning the AMBER architecture, we were aware of the constraint, and it probably had some influence on the final parameter settings. We hope that the heuristics discussed above will be of some aid in directing the search through the space of production system models. We also hope that the space defined by PRISM proves to contain the types of architectures that cognitive scientists find plausible and fertile. And while it is likely that many researchers will want to search beyond the space deline- 
ated by PRISM, we hope the formalism will have helped to clarify the dimensions along which production system architectures can vary and aided the quest for invariants of the human information processing system.

\section{REFERENCE NOTES}

1. Langley, P., \& Neches, R. Prism user's manual (Technical Report). Carnegie-Mellon University, Department of Computer Science, 1981 .

2. Rychener, M. Production systems as a programming language for artificial intelligence applications (Technical Report). Carnegie-Mellon University, Department of Computer Science, 1976.

3. Forgy, C. OPS4 user's manual (Technical Report). CarnegieMellon University, Department of Computer Science, 1979.

4. Forgy, C. On the efficient implementation of production systems (Technical Report). Carnegie-Mellon University, Department of Computer Science, 1979.

5. Lewis, C. Composition of productions. In D. Klahr, P. Langley, \& R. Neches (Eds.), Production system models of learning and development. Book submitted for publication, 1983.

\section{REFERENCES}

Anderson, J. R. Language, memory, and thought. Hillsdale, N.J: Erlbaum, 1976.

Anderson, J. R. Induction of augmented transition networks. Cognitive Science, 1977, 1, 125-157.

Anderson, J. R. The architecture of cognition. Cambridge, Mass: Harvard University Press, 1983.

Anderson, J. R., Kline, P. J., \& Beasley, C. M. Complex learning processes. In R. E. Snow, P. A. Federico, \& W. E. Montague (Eds.), Aptitude, learning, and instruction: Cognitive process analyses. Hillsdale, N.J: Erlbaum, 1980.

Anderson, J. R., KLine, P. J., \& Lewis, C. A production system model for language processing. In P. Carpenter \& $\mathrm{M}$. Just (Eds.), Cognitive processes in comprehension. Hillsdale, N.J: Erlbaum, 1977.

Anzal, Y. Learning strategies by computer. In Proceedings of the Second National Conference of the Canadian Society for Computational Studies of Intelligence, 1978.

HAYES-Roth, F. The role of partial and best matches in knowledge systems. In D. A. Waterman \& F. Hayes-Roth (Eds.), Pattern-directed inference systems. New York: Academic Press, 1978.

Hedrick, C. Learning production systems from examples. Artificial Intelligence, 1976, 7, 21-49.

Klaha, D., \& Wallace, J. G. Cognitive development: An information processing analysis. Hillsdale, N.J: Erlbaum, 1976.

KLINE, P. J. The superiority of relative criteria in partial matching and generalization. Proceedings of the Seventh International Joint Conference on Artificial Intelligence, 1981, 1, 296-303.

Kunn, T. S. International encyclopedia of unified science (Vol. 2, No. 2). The structure of scientific revolutions. Chicago: University of Chicago Press, 1962.

LANGLEY, P. Language acquisition through error recovery. Cognition and Brain Theory, 1982, 5, 211-255. (a)

Langley, P. A model of early syntactic development. In Proceedings of the 20th Annual Conference of the Society for Computational Linguistics, 1982. (b)

LANGLEY, P. Strategy acquisition governed by experimentation. In Proceedings of the European Conference on Artificial Intelligence, 1982. (c)

LANGLEY, P. Learning search strategies through discrimination. International Journal of Man-Machine Studies, in press.

McDermotT, J., \& Forgy, C. Production system conflict resolution strategies. In D. A. Waterman \& F. Hayes-Roth (Eds.),
Pattern-directed inference systems. New York: Academic Press, 1978.

Neches, R. Simulation systems for cognitive psychology. Behavior Research Methods \& Instrumentation, 1982, 14, 77-91.

Neves, D. M., \& Anderson, J. R. Knowledge compilation: Mechanisms for the automatization of cognitive skills. In J. R. Anderson (Ed.), Cognitive skills and their acquisition. Hillsdale, N.J: Erlbaum, 1981.

NEWELL, A. Production systems: Models of control structures. In W. C. Chase (Ed.), Visual information processing. New York: Academic Press, 1973.

NEWELL, A. Reasoning, problem solving, and decision processes: The problem space hypothesis. In R. Nickerson (Ed.), Attention and performance. Hillsdale, N.J: Erlbaum, 1980.

Newell, A., \& Simon, H. A. Human problem solving. Englewood Cliffs, N.J: Prentice-Hall, 1972.

Simon, H. A. Does scientific discovery have a logic? Philosophy of Science, 1973, 4, 471-480.

Simon, H. A., Langley, P., \& Bradshaw, G. Scientific discovery as problem solving. Synthese, 1981, 47, 1-27.

Waterman, D. A. Adaptive production systems. In Proceedings of the Fourth International Joint Conference on Artificial Intelligence, 1975.

Waugh, N. C., \& Norman, D. A. Primary memory. Psychological Review, 1965, 72, 89-104.

Young, R. M. Seriation by children: An artificial intelligence analysis of a Piagetian task. Basel: Birkhauser, 1976.

Young, R. M., \& O'Shea, T. Errors in children's subtraction. Cognitive Science, 1981, 5, 153-177.

\section{NOTES}

1. PRISM has been implemented on a DEC PDP-10 and a VAX-780. It will run on any operating system that supports either MACLISP or FRANZ LISP.

2. By convention, action side functions are enclosed in angle brackets $\langle$ and $>$. In specifying default actions, the symbol $x$ stands for the elements returned from a production's action side when it is applied. These elements are substituted for the $x$, and the default actions are evaluated. For example, if the default actions were $((<$ add $>x))$ and an action side returned the elements (want food) and (have food), then the action (<add $>$ (want food) (have food)) would be carried out, adding these two elements to working memory.

3. In some cases, the user may not wish to check on every cycle for efficiency reasons; PRISM also allows the user to specify how often this checking should occur.

4. Another approach to generalization involves comparing the condition sides of two rules and constructing a new rule that contains only those conditions that were held in common. Anderson et al. (1980), Hedrick (1976), and others have explored this approach to learning. PRISM does not support this version of generalization, largely because of its computational expense.

5. An alternate approach to discrimination involves the replacement of variables with constant terms. Anderson et al. (1980) have explored this approach to learning. PRISM does not incorporate this technique, since it can be adequately simulated using the condition-adding approach.

6. Since strong rules are preferred to weak ones, the system may go down a set of paths only to reach dead ends. Since states visited earlier are retained, in such cases SAGE would simply return to one or more states higher in the tree and continue the search down paths that had been ignored earlier.

7. In some sense, this space is infinite in size. For example, one may set the initial activation level of working memory elements to any real value, of which there are an infinite number. However, even ignoring options that take on numeric values, the set of possible combinations is quite large. 